\title{
Scaling near Quantum Chaos Border in Interacting Fermi Systems
}

\author{
Pil Hun Songt \\ Center for Theoretical Physics, Seoul National University, Seoul 151-742, Korea
}

(November 21, 2018)

\begin{abstract}
The emergence of quantum chaos for interacting Fermi systems is investigated by numerical calculation of the level spacing distribution $P(s)$ as a function of interaction strength $U$ and the excitation energy $\epsilon$ above the Fermi level. As $U$ increases, $P(s)$ undergoes a transition from Poissonian (nonchaotic) to Wigner-Dyson (chaotic) statistics and the transition is described by a single scaling parameter given by $Z=\left(U \epsilon^{\alpha}-u_{0}\right) \epsilon^{1 / 2 \nu}$, where $u_{0}$ is a constant. While the exponent $\alpha$, which determines the global change of the chaos border, is indecisive within a broad range of $0.9 \sim 2.0$, the finiteness of $\nu$, which comes from the increase of the Fock space size with $\epsilon$, suggests that the transition becomes sharp as $\epsilon$ increases.
\end{abstract}

PACS numbers: 05.45.-a, 05.30.Fk, 73.23.-b

Recently, the emergence of quantum chaos due to electron-electron interaction has attracted much attention. While the subject has a longer history in nuclear physics, with the progress of modern nanofabrication techniques, it has entered condensed matter physics since a quantum dot system, for example, could be considered as an artificial atom with some physical parameters under control. Besides being of interest as its own right, the importance of the subject stems from the fact that it is related to a failure of the perturbative approach in interacting many-particle systems. Traditionally, the perturbative method has been one of the standard tools in theoretical many-particle physics. The emergence of quantum chaos means a strong mixing of the unperturbed levels, thereby inducing breakdown of the perturbation series.

A recent theoretical work by Altshuler et al. [1] for the quasiparticle decay in a quantum dot has especially stimulated many theoretical investigations. In their paper [1], the quasiparticle decay process was mapped to a single particle diffusion on the Bethe lattice, which is a nonperturbative treatment of the problem. They concluded that there is a transition to quantum chaos at a critical excitation energy $\epsilon_{c} \sim \sqrt{g} \Delta$, where $g$ is the dimensionless conductance and $\Delta$ is the mean level spacing between the single-particle levels. However, many authors afterwards have pointed out that the mapping to a Bethe lattice in ref. [1] is too naive for a proper description and they obtained different results using other methods. The ongoing controversy could be summarised through two main questions. i) what is the relation between $\epsilon$ and $g$ at the quantum chaos border, and ii) whether the transition is sharp or not. Regarding the first question, there exist further different predictions such as $\epsilon_{c} \sim g^{2 / 3} \Delta$ [2,3] and $\epsilon_{c} \sim g \Delta / \ln g$ [A]. As for the second question, Jacquod and Shepelyansky [2] argued that the transition is smooth since the coupling between the Fock states is of nonlocal nature. The authors of ref. [5] concluded that the transition is smooth based on their numerical result for the local density of states and the participation number. In ref. [6], the same conclusion has been drawn by calculating the inverse participation ratio (IPR) for higher values of $\epsilon$ by use of the, so called, layer model. On the other hand, Berkovits and Avishai [7] suggested a finite size scaling hypothesis, according to which the transition becomes sharp as $\epsilon$ increases, which was based on their exact numerical results for small system size. Silvestrov [4] also proposed that the transition is sharp when the effective high-order interaction is taken into account. In addition, the authors of ref. [8] performed an analysis of their numerical result for the IPR and found that their results show features consistent with the prediction of ref. [4]. However, their conclusion is not decisive enough concerning the sharpness of the transition. A reliable numerical test is, therefore, urgently needed to settle the issue.

The main difficulty of numerical test is due to the fact that one should consider the regime of $g \gg 1$, which corresponds to $\epsilon \gg \Delta$. Since the size of the matrix to be diagonalized rapidly increases with $\epsilon$, one needs an alternative to the brute force method. The layer model, introduced by Georgeot and Shepelyansky [9], allows one to handle much larger system size (higher $\epsilon$ ) at a given computational cost by truncating out the Slater determinants which contribute little to a given eigenstate.

In this paper, we calculate the level spacing distribution $P(s)$ for interacting fermionic systems up to $\epsilon / \Delta=$ 27 by use of the layer model. The change of $P(s)$ from the Poissonian to Wigner-Dyson statistics represents the transition from integrability to chaos. While our result does not allow us to resolve the controversy over the parametric relation for the quantum chaos border, i.e. question (i), it gives strong evidence that the transition becomes sharper as $\epsilon$ increases. The finite size scaling (FSS) behaviour can be understood through a comparison with an infinite dimensional Anderson model.

Let us begin with $n_{f}$ spinless fermions on $m$ singleparticle levels for which the Hamiltonian $\mathcal{H}$ is given by $\mathcal{H}_{0}+\mathcal{H}_{1}$ with

$$
\mathcal{H}_{0}=\sum_{i} \epsilon_{i} c_{i}^{\dagger} c_{i} \text { and } \mathcal{H}_{1}=\sum_{i<j, k<l} U_{i j, k l} c_{l}^{\dagger} c_{k}^{\dagger} c_{i} c_{j} .
$$

$\mathcal{H}_{0}$ represents the noninteracting Hamiltonian. A single- 
particle level energy $\epsilon_{i}$ with $i=1,2, \cdots, m$ is randomly chosen from the interval $[(i-1 / 2) \Delta,(i+1 / 2) \Delta]$ with uniform probability distribution. $U_{i j, k l}$ denotes the random two-body interaction matrix element and also has a box distribution $[-U / 2, U / 2]$. When $\mathcal{H}$ is interpreted as the Hamiltonian for a quantum dot system, $U$ is related to $g$ as $U \sim \Delta / g$ [1, 10]. $\mathcal{H}$ is widely known as the TwoBody Random Interaction Model (TBRIM) and its full matrix size is given by $N_{t}=n_{f} ! /\left[m !\left(m-n_{f}\right) !\right]$. When $\mathcal{H}$ is written in matrix form on the basis of the eigenstates of $\mathcal{H}_{0}$, a diagonal element is the sum of the energies of occupied single-particle levels, while an off-diagonal element is nonzero only for two states which are different upto two particle-hole pairs.

Since $N_{t}$ increases very rapidly with $n_{f}$ and $m$, direct numerical diagonalisation of $\mathcal{H}$ is limited to rather small values of $n_{f}$ and $m$. Due to this difficulty, the layer model has been introduced by Georgeot and Shepelyansky [9] and the details are as follows. For each eigenstate of $\mathcal{H}_{0}$, we define a sequence $\left\{f_{i}\right\}$ such that $f_{i}=0$ when the level $i$ is empty and $f_{i}=1$ when it is filled with a fermion. Then $\mathcal{E} / \Delta=\sum_{i=1}^{m} i f_{i}$ ranges from $n_{f}\left(n_{f}+1\right) / 2$ to $n_{f}(m+1)-n_{f}\left(n_{f}+1\right) / 2$, which is approximately equal to the ground state energy and the highest excitation energy, respectively, in units of $\Delta$. If we rewrite $\mathcal{H}$ in ascending order of $\mathcal{E}$ and set $U_{i j, k l}=0$ for $i+j \neq k+l$, the whole matrix reduces to a block diagonal form where $\mathcal{E}$ is constant within each block. We call the submatrix with $\mathcal{E} / \Delta-n_{f}\left(n_{f}+1\right) / 2=j$ the $j$-th layer model. In general, the matrix size of the $j$-th layer model, $N(j)$, varies depending on $n_{f}$ and $m$. However, for $1 \ll n_{f} \ll m, N(j)$ for $j \leq n_{f}$ is determined solely by $j$ and behaves as 11 .

$$
N(j) \sim \exp (\pi \sqrt{2 j / 3}) / j
$$

The layer model hereafter is understood in such a sense. An eigenstate of $\mathcal{H}$ with energy $E$ can be written as a superposition of the eigenstates of $\mathcal{H}_{0}$, the energies of which lie within the width $\Gamma$ around $E$. $\Gamma$ is much less than $\Delta$, when $U \ll \Delta$, i.e. $g \gg 1$. This defines the valid regime of the layer model.

One of the well established criteria for transition from integrability to chaos is the change of the level spacing distribution $P(s)$ from the Poissonian $P_{p}(s)=e^{-s}$ to the Wigner surmise $P_{w}(s)=(\pi s / 2) e^{-\pi s^{2} / 4}$. To quantify the proximity of $P(s)$ to either of the two, it is useful to define $\eta$ in terms of the variance of $s$ as follows;

$$
\eta=\frac{\int_{0}^{\infty} s^{2}\left(P(s)-P_{w}(s)\right) d s}{\int_{0}^{\infty} s^{2}\left(P_{p}(s)-P_{w}(s)\right) d s} .
$$

In this way, $\eta$ is 1 for $P_{p}(s), 0$ for $P_{w}(s)$ and inbetween for an intermediate distribution.

In our calculation, the Hamiltonian for the layer model for $19 \leq j \leq 27$ has been constructed and numerical diagonalisation has been performed over 200-1000 disorder configurations for each parameter set $(j, g)$. Corresponding matrix size ranges from $490(j=19)$ to 3010 $(j=27)$. To exclude the contribution of the tail states near the edge, $50 \%$ of eigenvalues around the band center have been used to construct $P(s)$. If we choose a smaller part of the eigenvalue set, e.g. $25 \%$, the result does not change significantly.

The $\eta$ 's for $19 \leq j \leq 27$ and for $1 / 200 \leq U \leq 1 / 10$ 112 are shown in Fig. 1. The gradual change from $\eta \simeq 1$ (Poissonian, integrable) to $\eta \simeq 0$ (Wigner-Dyson, chaotic) with increase of $U$ is found with all $j$. As $j$ increases, the transition occurs at a smaller value of $U$, indicating that the interaction becomes more efficient for mixing the levels as the energy increases. The global dependence of the chaos border on the excitation energy may be found by plotting the data of Fig. 1 on a rescaled $x$-axis with $x=U j^{\alpha}[13]$. If there exists $\alpha=\alpha_{0}$ such that all the data points lie in the same curve, the transition is described by the relation $\epsilon=U^{-1 / \alpha_{0}}$ and there is a smooth crossover instead of a sharp phase transition when $\epsilon \rightarrow \infty$. However there does not exist such a value $\alpha_{0}$ for our data. Instead, as shown in Fig. 2(a-c), there is a single crossing point where the curves meet one another and the slope at the crossing point becomes larger as $j$ increases. This suggests that at sufficiently high energy the transition to chaos is a sharp phase transition. In fact such a FSS feature with $j$ is found for a broad range of $0.9 \leq \alpha \leq 2$. When $\alpha$ is tuned from 0.9 to $2, \eta_{c}$ ( $\eta$ at the crossing point) decreases monotonically from 0.8 to 0.2 . For $\alpha<0.9$ or $\alpha>2$, the crossing point cannot be clearly identified. These results imply that while we cannot determine the exponent $\alpha$ which governs the quantum chaos border [14], the transition shows a FSS property regardless of the choice of $\alpha$ as long as $0.2<\eta_{c}<0.8$.

For a further analysis of this FSS behaviour, we assume that $\eta$ is given by a function $f$ of a single scaling variable $Z$ such that

$$
\eta(\epsilon, U)=f(Z)=f\left[\left(U \epsilon^{\alpha}-u_{0}\right) \epsilon^{1 / 2 \nu}\right]
$$

where $u_{0}$ is a constant of the order of unity which is independent of $U$ and $\epsilon$. To understand the introduction of this scaling parameter $Z$, it would be helpful to compare the layer model with an Anderson Hamiltonian. One can think of the standard Anderson Hamiltonian defined on a graph, which has the same structure as the $j$-th layer model; there are $N(j)$ vertices and a bond between two vertices exists if two corresponding Slater determinants of the $j$-th layer model have nonzero transition amplitude by the two-body interaction. The size of the graph can then be defined by the average minimum number of bonds between two vertices. It is given by $\sim \sqrt{j}$, which is the same as the typical number of electron-hole pairs for the $j$-th layer model [3]. The spatial dimension of the graph in the limit of $j \rightarrow \infty$ is given by

$$
d=\lim _{j \rightarrow \infty} \frac{\ln N(j)}{\ln (\sqrt{j})} \rightarrow \infty .
$$


We expect that there exists a transition to chaos as we decrease the disorder strength of the on-site energy for a fixed value of hopping energy between nearest neighbors. The transition will show a FSS behaviour with the system size $L \sim j^{1 / 2}$ and with $\nu=1 / 2$, the correlation length exponent of infinite dimensional Anderson model [15]. Returning back to the layer model, we expect that a similar FSS property exists since there also is the competition between the system size and a correlation length governing the chaotic property of the system. A difference between the layer models and the thus defined Anderson models is that the matrix elements are strongly correlated in the former, while they are completely random in the latter 16]. Therefore, $\nu$ of Eq. (4) is not necessarily equal to that of the infinite Anderson model, $1 / 2$. In passing, we note that Berkovits and Avishai [7] introduced a scaling hypothesis very similar to Eq. (4) based on their numerical results for lower energy.

According to Eq. (4), the slope of the curves $\eta(U)$ for a given $j$ behaves as $\sim j^{\alpha+1 /(2 \nu)}$ at the crossing point and $\nu$ is obtained by fitting this variation of the slopes for a given value of $\alpha$. Fig. 3 shows the scaling plot obtained in this way with $\alpha=1.5\left(\eta_{c}=0.56\right)$ and in this case $\nu$ is given by 0.59 . Such a procedure can be performed in the same way for $0.2 \leq \eta_{c} \leq 0.8(0.9 \leq \alpha \leq 2)$ and the result for $1 /(2 \nu)$ is shown in Fig. 4. Taking account of uncertainties of all data, $1 /(2 \nu)$ lies between 0.5 and $1.5(0.3<\nu<1)$ over the whole range where the crossing point is identified. Therefore, though $\nu$ also varies in a rather broad interval, our data evidently excludes the possibility of $\nu$ being infinity, i.e. the possibility of a smooth crossover. One should note that this large variation in $\nu$ over a factor of 3 is the result of the fact that $\alpha$ is indecisive. If one can pin down the range of $\alpha$ in some other way than our method, or assume one of the previously proposed values (e.g. 1.5 [2, 3] or 2 [1]) to be valid, the uncertainty in $\nu$ can be determined to a higher accuracy from Fig. 4.

Finally, we discuss the sharpness of the transition when $\epsilon$ is varied for a fixed value of $U$, since in real measurements with a quantum dot the conductance of the sample $g \sim \Delta / U$ is kept constant and the bias voltage, which corresponds to $\epsilon$, is varied. We define the transition as sharp when the ratio of the transition interval $\delta \epsilon$ to the transition energy $\epsilon_{c}$ goes to zero as $\epsilon_{c}$ increases. From Eq. (4), $\epsilon_{c}$ is given by $\left(u_{0} / U\right)^{1 / \alpha}$ and $\delta \epsilon$ is estimated using the slope of the curve $\eta(\epsilon, U)$ as

$$
\delta \epsilon \sim\left|\frac{\partial \eta}{\partial \epsilon}\right|_{\epsilon=\epsilon_{c}}^{-1} \sim \epsilon_{c}^{1-\frac{1}{2 \nu}} .
$$

Therefore, $\left.(\delta \epsilon / \epsilon)\right|_{\epsilon=\epsilon_{c}} \sim \epsilon_{c}^{-1 /(2 \nu)}$ and we find that the transition becomes sharp as long as $\nu$ is not infinity. Therefore, the result of Fig. 4 shows that the transition to chaos takes place sharply if one uses a sufficiently clean sample, i.e. large $g$, for a quantum dot.

In summary, we have numerically investigated the emergence of quantum chaos in the interacting many- fermion systems using the layer model. While our result does not allow us to find the global dependence of the chaos border as a function of the interaction strength, we find that the transition becomes sharp at sufficiently high energy. This is also true when one observes the transition to chaos in a quantum dot as a function of bias voltage.

I am thankful to Jae Dong Noh, Gun Sang Jeon and especially to Dima L. Shepelyansky for helpful discussions.

* present address: Max-Planck-Institut für Physik komplexer Systeme, Nöthnitzer Str. 38, D-01187 Dresden, Germany, Email: phsong@mpipks-dresden.mpg.de

[1] B. L. Altshuler, Yu. Gefen, A. Kamenev and L. S. Levitov, Phys. Rev. Lett. 78, 2803 (1997).

[2] P. Jacquod and D. L. Shepelyansky, Phys. Rev. Lett. 79, 1837 (1997).

[3] A. Mirlin and Y. Fyodorov, Phys. Rev. B 5613393 (1997).

[4] P. G. Silvestrov, Phys. Rev. E 58, 5629 (1998).

[5] C. Mejia-Monasterio, J. Richert, T. Rupp, and H. A. Weidenmüller, Phys. Rev. Lett. 815189 (1998).

[6] X. Leyronas, J. Tworzydlo, and C. W. Beenakker, Phys. Rev. Lett. 82, 4894 (1999).

[7] R. Berkovits and Y. Avishai, Phys. Rev. Lett. 80, 568 (1998).

[8] X. Leyronas, P. G. Silvestrov and C. W. Beenakker, Phys. Rev. Lett. 843414 (2000).

[9] B. Georgeot and D. L. Shepelyansky, Phys. Rev. Lett. 79 4365 (1997).

[10] Ya. M. Blanter, Phys. Rev. B 54, 12807 (1996).

[11] A. Bohr and B. R. Mottelson, Nuclear Structure (Benjamin, New York, 1969), Vol. 1, p. 284.

[12] We may set $\Delta=1$ without loss of generality. Under this condition, the relations between parameters become simpler to yield $g \sim 1 / U$ and $\epsilon=j$, for example.

[13] We note that within the finite range of $1 / 200 \leq U \leq 1 / 10$ $(10 \leq g \leq 200)$, the functional form of $g / \log g$, which was

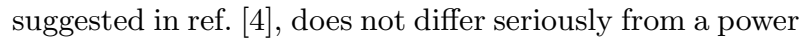
form of $g^{1 / \alpha}$.

[14] We may try a more refined analysis through higher cumulants of $P(s)$ assuming that just at the transition point, $P(s)$ will be given by a critical form $P_{c}(s)$ which is insensitive to $U$ and $j$. Unfortunately, such an approach is not successful within our data accuracy.

[15] K. B. Efetov, Physica (Amsterdam) 167A, 119 (1990).

[16] For example, the correlation of the diagonal energies for the layer model decays very slowly with the distance as $\left\langle\mathcal{H}_{i i} \mathcal{H}_{j j}\right\rangle \sim C-d(i, j)$, where $C$ is a constant of order of $n_{f}$ and $d(i, j)$ is the distance between two Slater determinants or two vertices characterized by $i$ and $j$ respectively. In contrast, it is zero unless $i=j$ for the Anderson models. 


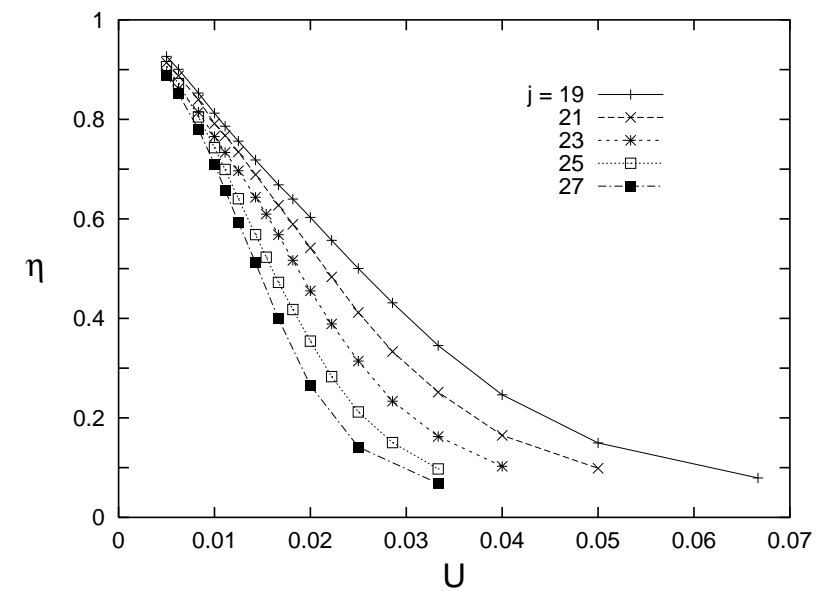

FIG. 1. $\eta$ as a function of the interaction strength $U$ for the layer model with various values of $j$.
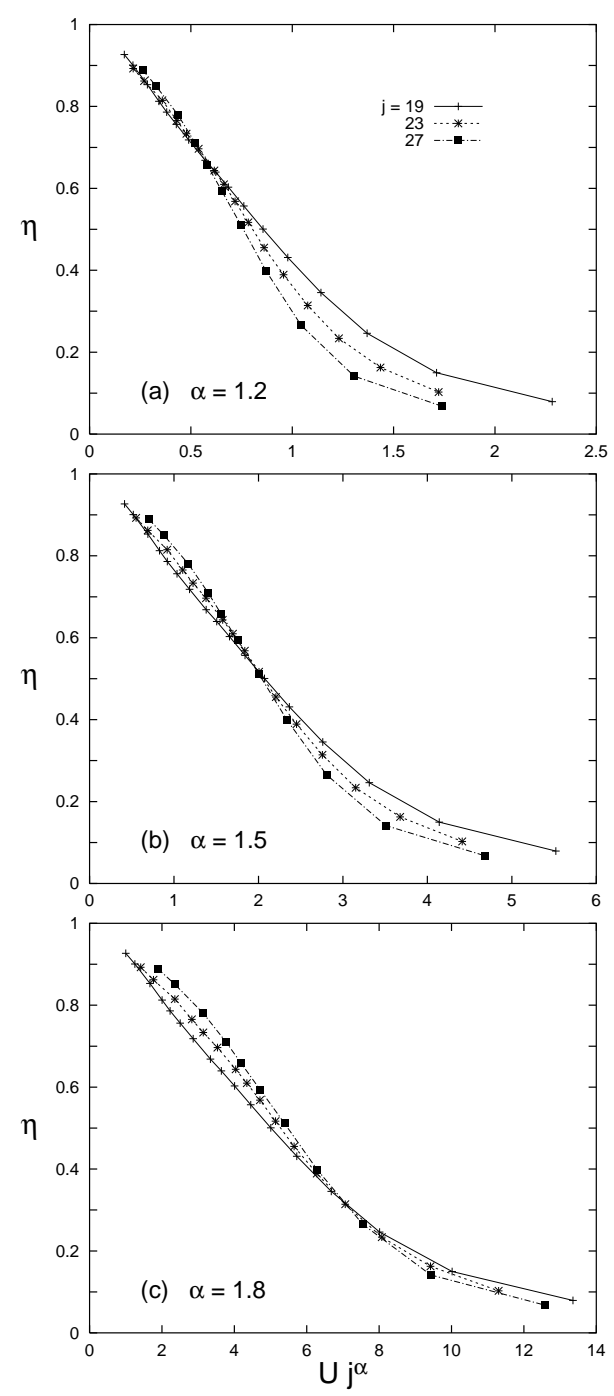

FIG. 2. The same data as Fig. 1 with rescaled $x$-axis of $x=U j^{\alpha}$ with $\alpha=1.2,1.5$ and 1.8. A partial set of data from Fig. 1 is shown for clarity.

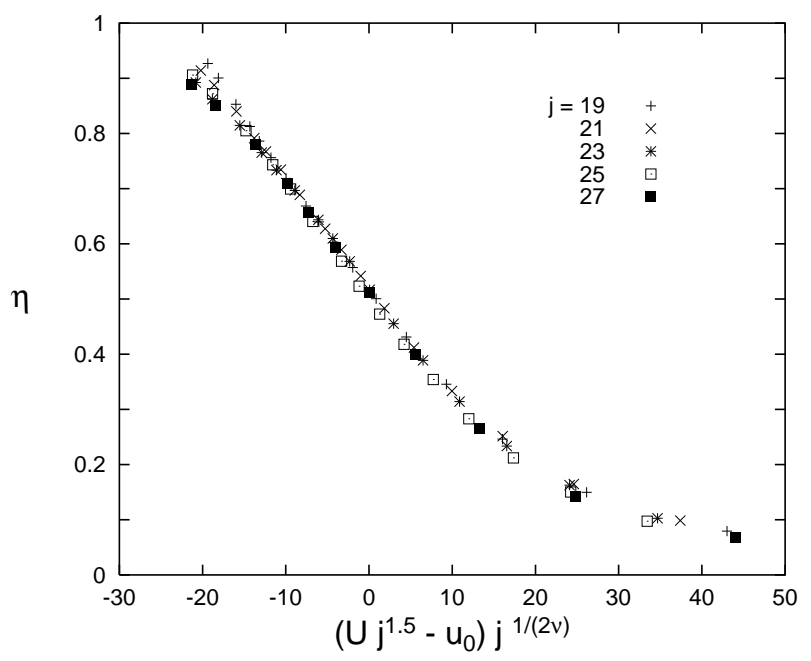

FIG. 3. Scaling plot of the data of Fig. 1 for $\alpha=1.5$. In this case $1 /(2 \nu)$ is given by 0.85 , i.e. $\nu=0.59$.

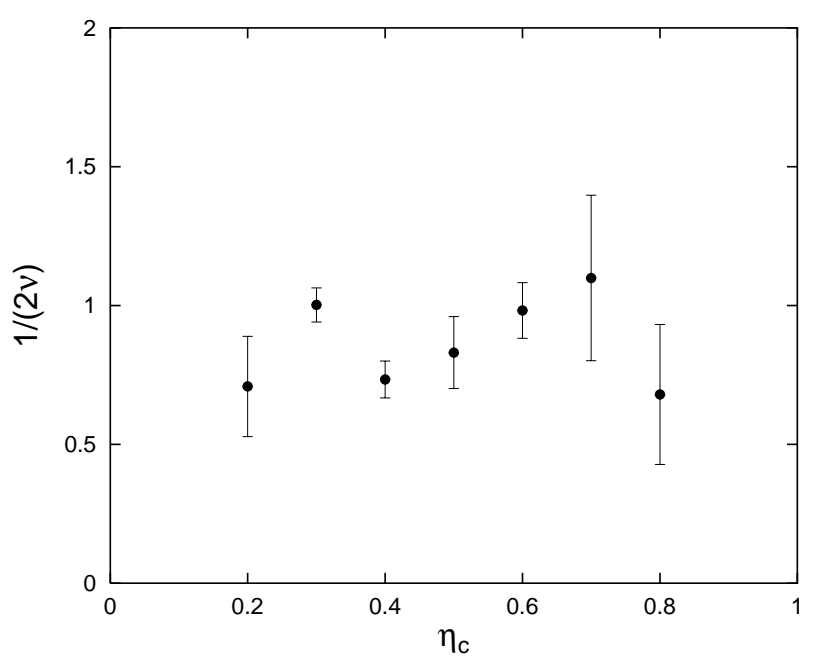

FIG. 4. $1 /(2 \nu)$ when $\eta_{c}$ varies from 0.2 to 0.8 